\title{
Protocol for Systematic Literature Review of Face Recognition in Uncontrolled Environment
}

\author{
Faizan Ullah, Sabir Shah, Dilawar Shah, Abdusalam, Shujaat Ali \\ Department of Computer Science, Bacha Khan University, Charsadda, KPK, Pakistan \\ faizanullah@bkuc.edu.pk
}

\section{Abstract}

\begin{abstract}
One of the major challenges encountered by current face recognition techniques lies in the difficulties of handling varying poses, i.e., recognition of faces in arbitrary in-depth rotations. The face image differences caused by rotations are often larger than the inter-person differences used in distinguishing identities. Face recognition across pose, on the other hand, has great potentials in many applications dealing with uncooperative subjects, in which the full power of face recognition being a passive biometric technique can be implemented and utilized. Extensive efforts have been put into the research toward pose-invariant face recognition in recent years and many prominent approaches have been proposed. However, several issues in face recognition across pose still remain open, such as lack of understanding about subspaces of pose variant images, problem intractability in 3D face modelling, complex face surface reflection mechanism, etc. This paper provides a critical survey of researches on image-based face recognition across pose. The existing techniques are comprehensively reviewed and discussed. They are classified into different categories according to their methodologies in handling pose variations. Their strategies, advantages/disadvantages and performances are elaborated. By generalizing different tactics in handling pose variations and evaluating their performances, several promising.
\end{abstract}

Keywords: Face recognition, illumination, pose, facial expression .

Received on 23 October 2017, accepted on 04 January 2018, published on 13 April 2018

Copyright (C) 2018 Author et al., licensed to EAI. This is an open access article distributed under the terms of the Creative Commons Attribution licence (http://creativecommons.org/licenses/by/3.0/), which permits unlimited use, distribution and reproduction in any medium so long as the original work is properly cited.

doi: 10.4108/eai.13-4-2018.154477

\section{Introduction}

The inspiration behind automatic face recognition is to provide the computer the similar capability as human beings to recognize faces. The general definition to face recognition is to estimate the identity of one or more people from static images or video sequences using a stored database of gallery faces. There are two types of face recognition: closed set and open set. In closed-set recognition, we can definitely find a gallery face matching the input image. In open-set recognition, we might not find any matched gallery image to the input face image. In the past decade's people focused on developing fundamental face recognition algorithms [1] [2] based on controlled environments which have simple backgrounds and limited variation in pose and lighting. To compare the performance of face recognition algorithms, a number of standard face databases were published, for example FERET [4], XM2VTS [3] and Multi-PIE [5]. Images from controlled databases are illustrated in Figure 1.2a. After years of development many proposed face recognition algorithms have produced very impressive results in these controlled databases. In the past, face recognition algorithms were evaluated on controlled face databases in which images have simple backgrounds and limited variation in pose and lighting. From left to right, example images are from XM2VTS [3], Yale[6], MultiPIE [5] and FERET [4] face databases respectively. b) Recently research has shifted to face recognition in uncontrolled environments in which images have complex backgrounds, partial occlusions and large variations in pose, lighting and race. Example images are from the most famous uncontrolled face database: Labeled Faces in the Wild [7]. Recently, research has shifted toward face recognition in uncontrolled environments to encourage 
real-world applications. Images are collected from the internet and have complex backgrounds, partial occlusion and large variations in pose, lighting, image quality, race and expression. The most famous uncontrolled face database is the Labeled Faces in the Wild database with over 600 citations in the face recognition literature [8]. Examples from this database are shown in Figure 1.2b. An ideal face recognition algorithm should perform well in uncontrolled environments to satisfy the requirements of real-world applications. However, this still remains a big challenge for most current face recognition algorithms. We would like to draw your attention to the fact that it is not possible to modify a paper in any way, once it has been published. This applies to both the printed book and the online version of the publication. Every detail, including the order of the names of the authors, should be checked before the paper is sent to the Volume Editors.

\subsection{Challenges of Uncontrolled Environments}

The three main challenges for face recognition in uncontrolled environments are large variation in pose, lighting and partial occlusion. The first challenge is pose variation. A person appears very differently from different viewpoints (see Figure 1.3a). Pose variations make the feature matching between two face images under different pose very difficult. In general, non-matching frontal faces are more similar to each other in terms of pixel values than matching faces of different poses. A second major obstacle is lighting variation (Figure 1.3b). It is hard to recognize the face under varying lighting. Even two images from the same person but under different lighting can appear dramatically different a)

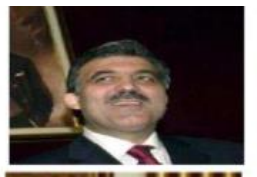

b)

c)

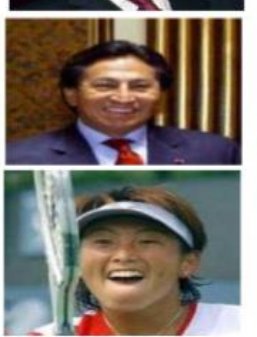

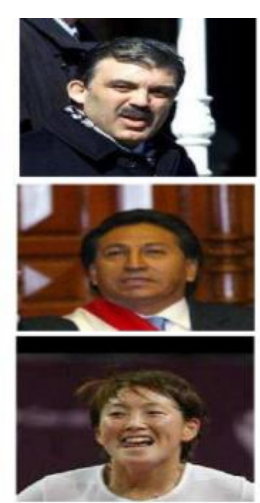

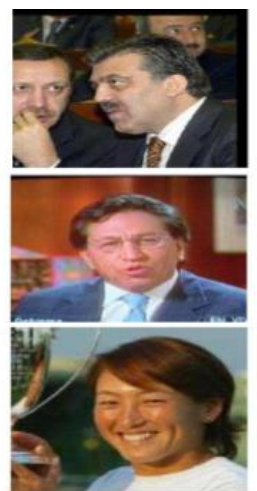

\section{Research Questions:}

Design of research questions is the most essential part of any systematic review [12]. It is very important to ask the true questions that are significant and can determine and/or scope future research activities [12]. The research questions have been design with the aid of PICOC standards in this systematic literature review as suggested by Petticrew and Roberts [14]. The main objective of systematic review is not to find any proof about comparison of methods/approach/model, so that why we ignore 'Comparison' in PICOC model.

\begin{tabular}{|l|l|}
\hline Population & $\begin{array}{l}\text { Face recognition, Facial recognition, Face } \\
\text { verification, Face classification, person identification }\end{array}$ \\
\hline Intervention & System, algorithms, Method, technique, \\
\hline Outcome & Fast, accurate \\
\hline Context & $\begin{array}{l}\text { This step will be broad. It will include academia as } \\
\text { well as software industry and all types of } \\
\text { stakeholders (developers, maintainers, testers, } \\
\text { project managers, students, researchers etc.) }\end{array}$ \\
\hline
\end{tabular}

In order to gather the proof of the research on the current state of face recognition in uncontrolled environment, the bellow research questions we have considered.

Q1. What are the open research challenges of face recognition in uncontrolled environment?

Q2. What evidences is there for face recognition from video surveillance?

Q3. What are the effective techniques for human face recognition from video in uncontrolled environment?

Q4. How recognition rate can be improved by analyzing different parameters?

\section{Search Strategy}

\subsection{Strategy for deriving search}

The scheme of search to derive search terms is given below. Some search term derived from search questions by identifying population, intervention, outcome and context table 2

\begin{tabular}{|l|l|}
\hline Population & Face recognition \\
\hline Intervention & Method, technique, system, algorithms \\
\hline Outcome & Robust, accuracy rate \\
\hline Context & Security, surveillance, real world \\
\hline
\end{tabular}

Search different keywords in relevant paper, table 3. Note that the studies from which we have taken the key words are not a subset of our primary studies. Rather they are taken from the literature relevant to the disciplines of face recognition in uncontrolled environment

\begin{tabular}{|l|l|}
\hline J. Phillips(1998) & $\begin{array}{l}\text { Face Recognition ; K-Means; SVM ;Spoof Detection; } \\
\text { KSVM }\end{array}$ \\
\hline Mehran Kafai(2015) & $\begin{array}{l}\text { Face, Probes, Face recognition, Databases, Histograms, } \\
\text { Feature extraction, Image recognition }\end{array}$ \\
\hline Xiaoying Wang(2016) & $\begin{array}{l}\text { Face recognition, low-resolution (LR), pose variations, } \\
\text { discriminant analysis }\end{array}$ \\
\hline Xu Zhou(2016) & $\begin{array}{l}\text { Face, Training, Detectors, Principal component analysis, } \\
\text { Face detection, Face recognition, Image resolution }\end{array}$ \\
\hline Vijayalakshmi(2015) & $\begin{array}{l}\text { Video based face recognition, face recognition, occlusion, } \\
\text { pose variation, Discrete Curvelet Transform }\end{array}$ \\
\hline Simon Dobrišek (2015) & $\begin{array}{l}\text { Image classification, face recognition, feature extraction, } \\
\text { Gabor filters }\end{array}$ \\
\hline \multicolumn{2}{|c|}{ Table 3: Key words from relevant papers }
\end{tabular}

To make the search terms and the synonyms and alternate spellings with the help of a thesaurus. Also mention if a subject librarian and/or content experts in the field are consulted, table 4 . Note that the terms in italics have been added from table 3 . 


\begin{tabular}{|c|c|}
\hline Face recognition & $\begin{array}{l}\text { Facial recognition, Face verification, Face classification, } \\
\text { person identification }\end{array}$ \\
\hline Method & System, technique, process, procedure \\
\hline Real world & $\begin{array}{l}\text { Unconstrained environment, video surveillance, real world } \\
\text { video }\end{array}$ \\
\hline \multicolumn{2}{|c|}{$\begin{array}{l}\text { Use Boolean OR to construct search strings from the search terms identified in (i), } \\
\text { (ii), and (iii), (see table 5). }\end{array}$} \\
\hline \multicolumn{2}{|c|}{$\begin{array}{l}\text { Face recognition OR Facial recognition OR Face verification OR Face classification } \\
\text { OR Person Identification }\end{array}$} \\
\hline Method OR System & $R$ technique OR process OR procedure \\
\hline
\end{tabular}

\begin{tabular}{|l|}
\hline $\begin{array}{l}\text { Real world OR Unconstrained environment OR video surveillance OR real world } \\
\text { video }\end{array}$ \\
\hline \multicolumn{2}{c}{ Table 5 Construction of Terms by using Boolean OR } \\
\hline
\end{tabular} Use Boolean AND to concatenate the search terms and restrict the research, (see table $6)$.

(Face recognition OR Facial recognition OR Face verification OR Face classification) AND ( Method OR System OR technique OR process OR procedure) AND (Robust OR Fast OR accurate OR wild) AND (Real world OR Unconstrained environment OR video surveillance OR real world video)

Table 6: Concatenation of Terms by Using Boolean AND

\subsection{Search Process and Resources}

Searching method for a systematic review must be rigorous and be able to discover as many relevant primary studies as possible [12] [13]. This systematic literature will take to two search phases Secondary search phase and initial/primary to make the search process rigorous.

\section{2.1 Primary Search Phase}

Primary search phase will be applied on online database, conference proceedings, grey literature (such as $\mathrm{MSc} / \mathrm{PhD}$ theses and dissertation and technical reports) electric journals, search engines. To make searching process easy and evade the risks of missing out evidence, our search will be consisting of literature published in 2008 to date. 2008 will be the starting edge due to the reason that we apply some random search on face recognition in uncontrolled environment before 2008 and the result we found are zero. This phase provides a details information of electronic search. The detail list is given below.

\begin{tabular}{|c|c|c|c|}
\hline \multicolumn{4}{|l|}{ Online databases } \\
\hline - Science Direct & $\bullet$ & \multicolumn{2}{|c|}{ ACM Digital Library } \\
\hline - $\quad$ ProQuest & $\bullet$ & \multicolumn{2}{|c|}{ SCOPUS } \\
\hline \multicolumn{4}{|l|}{ Online Search engines: } \\
\hline $\begin{array}{lll}- & \begin{array}{l}\text { Google } \\
\text { (scholar.google.com) }\end{array} \\
\end{array}$ & & \multicolumn{2}{|c|}{ Cite Seer (citeseer.ist.psu.edu) } \\
\hline \multicolumn{4}{|l|}{ Journals: } \\
\hline - $\quad$ Springer & & $\begin{array}{l}\text { Machine } \\
\text { Applications }\end{array}$ & Vision and \\
\hline $\begin{array}{l}\text { International Journal } \\
\text { of Computer Vision }\end{array}$ & & $\begin{array}{l}\text { International } \\
\text { Multimedia } \\
\text { Retrieval }\end{array}$ & $\begin{array}{l}\text { Journal of } \\
\text { Information }\end{array}$ \\
\hline
\end{tabular}

\subsection{Secondary Search Stage}

Secondary search phase will be conducted to supplement the primary search stage. The below actions will be achieved in this stage.
1. References of studies will be identified in primary stage will be studied. This procedure will be an iterative unless the relevant paper is included to list of primary articles and the same step will be applied on each articles.

2. Citations of the articles will be studied for the identification of primary studies and the studies citing the identified primary studies will also be reviewed by using backward and forward passes (as also suggested by Webster \& Watson [3]). This process is also iterative in nature.

3. Individual researcher on relevant area will be identified and communicated for advice on unpublished work and technical reports.

\subsubsection{Search Process Documentation}

Documenting the search process provides transparency, helps prevent bias effect, provides details of thoroughness, and enables replication of the search process. The systematic review will follow the documentation procedures provided by Kitchenham [1], (see table 7).

\begin{tabular}{|l|l|}
\hline Data Source & Documentation \\
\hline Online Databases/Digital Libraries & $\begin{array}{l}\text { Name of database } \\
\text { Search strategy for database } \\
\text { Date of search } \\
\text { Years covered by search }\end{array}$ \\
\hline Search Engine & $\begin{array}{l}\text { Name of search engine } \\
\text { Search strategy for the search engine } \\
\text { Date of search }\end{array}$ \\
\hline Journal Hand Searches & $\begin{array}{l}\text { Journal name } \\
\text { Years covered } \\
\text { Any issues not covered }\end{array}$ \\
\hline Conference Proceedings & $\begin{array}{l}\text { Title of Proceedings } \\
\text { Name of Conference (if different) } \\
\text { Journal name (if published in a journal) } \\
\text { Conference Date }\end{array}$ \\
\hline PhD Dissertations and Theses & $\begin{array}{l}\text { Title of dissertation/thesis } \\
\text { Publication Year } \\
\\
\text { Name of University } \\
\text { URL (if available) }\end{array}$ \\
\hline Unpublished studies & $\begin{array}{l}\text { Research groups and researchers contacted } \\
\text { (contact information and date contacted) } \\
\text { Research web sites searched (URL and Date) }\end{array}$ \\
\hline Other sources & $\begin{array}{l}\text { Date Searched/contacted } \\
\text { URL }\end{array}$ \\
\hline & \\
\hline
\end{tabular}

Table 7 Documentation of Search Process

\subsection{Quality Assessment Criteria and Selection Process}

Motivation behind this study is to find relevant paper which provide proof about the research question [1]. Quality assessment will be applied to find relevance's between selected study and research questions, but the selection criteria will be on the base of strong evidence which provide answer to the research questions. If there is any study which does not fall in above criteria will be excluded.

\subsubsection{Rejection/Selection Criteria for Study Selection}

The selection and rejection criteria will be enhanced during search process. For a study to include in systematic literature review it will have to satisfy the below three conditions. 
a) The articles having Face Recognition (any face classification/facial recognition and or types Face Recognition) in uncontrolled environment

b) The articles having discusses approach/techniques for face recognition in uncontrolled environment.

c) The articles which having experimental results of findings.

It is to be noted that there is no restriction that the articles belong to which groups or individual, either they are student, researcher or experienced practitioners. The selection criteria are purely based on answer to research questions. The articles which have the below characteristics will be excluded from systematic review.

a) Where the focus is on controlled environment other than uncontrolled environment.

b) Where the study does not perform an experimental validation of the findings that it presents.

\subsubsection{Preliminary/Initial Selection Phase}

During the preliminary selection Phase abstract and title of the paper will be studied of selected articles if they satisfy the maximum threshold criteria will be included for detail study otherwise the paper will be discarded.

\subsubsection{Final Selection Phase}

During selection stage if it is not clear that the articles meet selection criteria or not, get its hard copy and read the full paper. During this reading if the paper meet relevant to study will be included to final reference library. If there any confusion in the articles will discussed with colleagues and supervisor.

\section{Quality Analysis Checklists and Measures}

For analysis of quality study and checklist for quantitative and qualitative study had been defined. We have defined checklist individually for quantitative and qualitative studies, because the research methodologies are different for both. Separate Checklist are very important as it help to conceive detailed questions. The main purpose of checklist is to measure the quality of research articles as evidence to response a research question, while the purpose of checklist is not criticizing any research work. Example of some questions are found in checklist found by $\mathrm{C}$ rombie [7], Fink [8], and Petticrew [9]. Some questions for systematic literature are present in Kitchenham's checklists [1]. Value to checklist are given in table 9 and table 10 and each of question of qualitative and quantitative will be answered in table 8 .

\begin{tabular}{|l|l|}
\hline Answer & Score \\
\hline Yes & 1 \\
\hline No & 0 \\
\hline Partially & 0.5 \\
\hline
\end{tabular}

Table 8 weight for answering questions in quality checklists

The weightage score for quantitative study will be in between 0 to 18,18 represent very high quality and 0 represent very low quality articles.

\section{Quantitative Checklists}

The quantitative study contains a set of 18 question which will be used to analysis the quantitative studies (see table 9).

\begin{tabular}{|c|c|c|}
\hline Sr. No. & Que & Answer \\
\hline 1 & Are the research question(s) clearly stated for the article? $[7,8]$ & Yes/No/Partially \\
\hline 2 & $\begin{array}{l}\text { Do the articles based on existing body, like does it clearly discuss its } \\
\text { research in light of literature? }\end{array}$ & Yes No/Partially \\
\hline 3 & $\begin{array}{l}\text { Is the features metrics applied in article sufficiently measured and } \\
\text { tested[7,8,9] }\end{array}$ & Yes No/Partially \\
\hline 4 & Is the database used in the article clearly defined? [8] & Yes No/Partially \\
\hline 5 & Are all proposed model/system are defined (methods and tool used)? & Yes No/Partially \\
\hline 6 & Are the data source collection methods sufficiently described? [8] & Yes No/Partially \\
\hline 7 & Are the statistical methods reasonable? [8] & Yes No/Partially \\
\hline 8 & Is the purpose of the data analysis clear? [8] & Yes/No/Partially \\
\hline 9 & Is the study contains hybrids techniques? & Yes No Partially \\
\hline 10 & Are the negative results presented? $[8]$ & Yes No Partially \\
\hline 11 & $\begin{array}{l}\text { Do the researchers discuss any problems with the validity reliability of } \\
\text { their results? [8] }\end{array}$ & YesNo/Partially \\
\hline 12 & Is the study repeatable? & Yes/No/Partially \\
\hline 13 & Is the research design clearly presented? & Yes No Partially \\
\hline 14 & Is the research design suitable for carrying out the study? $[7,8,9]$ & Yes/No/Partially \\
\hline 15 & Are the findings credible? [9] & YesNo Partially \\
\hline 16 & $\begin{array}{l}\text { Are the data flow elearly presented, from initial stage to final stage of } \\
\text { recognition? }\end{array}$ & Yes No/Partially \\
\hline 17 & Are the links between the data, interpretation, and conclusions clear? & Yes No/Partially \\
\hline 18 & Is the reporting clear and coherent? & Yes No/Partially \\
\hline
\end{tabular}

\subsection{Qualitative Check Lists}

The qualitative checklist consists of 8 questions to be used for the evaluation of qualitative studies, (see table 10). Note that item 2 has been adapted from [9].

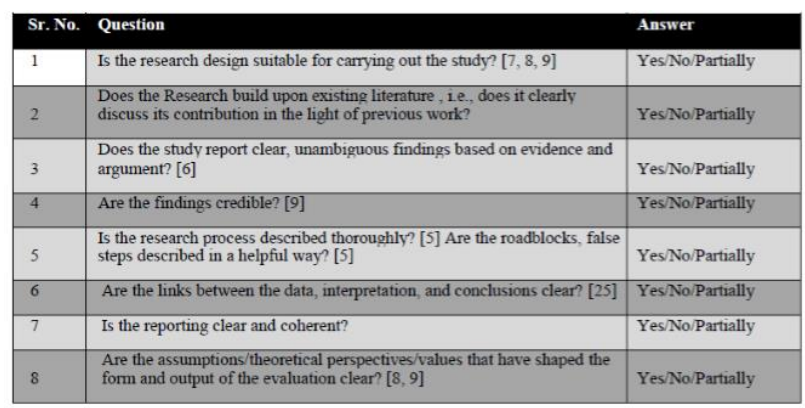

Table 10 Checklist for qualitative studies 


\section{References}

1. M. Turk and A. Pentland. Eigenfaces for recognition. Journal of Cognitive Neuroscience, 3:71-86, 1991.

2. P. Belhumeur, J. Hespanha, and D. Kriegman. Eigenfaces vs. Fisherfaces: recognition using class Specific linearprojection. IEEE Transactions on pattern analysis and machine intelligence, 19(7):711-720, 1997.

3. K.Messer, J. Matas, J. Kittler, J. Luettin, and G.Maitre. Xm2vtsdb: The extended m2vts database. In SecondInternational Conference on Audio and Videobased Biometric Person Authentication, 1999.

4. P. Phillips, H. Wechsler, J. Huang, and P. Rauss. The FERET database and evaluation procedure for face recognition algorithms. Image and Vision Computing, 16(5):295-306, 1998. 5. R. Gross, I.Matthews, J. Cohn, T. Kanade, and S. Baker. Multi-pie. Image and Vision Computing, 28(5):807-813, 2010.

6. A. S. Georghiades, P. N. Belhumeur, and D. Kriegman. From few to many: Illumination cone models for face recognition under variable lighting and pose. IEEE Transactions on Pattern Analysis and Machine Intelligence, 23(6):643-660, 2001.

7. G. B. Huang, M. Ramesh, T. Berg, and E. LearnedMiller. Labeled faces in the wild: A database for studying face recognition in unconstrained environments. Technical Report 07-49, University of Massachusetts, Amherst, October 2007.

8. Y. Taigman and L. Wolf. Leveraging billions of faces to overcome performance barriers in unconstrained face recognition. arXiv preprint arXiv:1108.1122, 2011.

9. T. Berg and P. N. Belhumeur. Tom-vs-pete classifiers and identity-preserving alignment for face verification. In British Machine Vision Conference, volume 1, page 5, 2012.

10. M. Belkin and P. Niyogi. Laplacian eigenmaps and spectral techniques for embedding and clustering. Advances in neural information processing systems, 14:585-591, 2001.

11. D. J. Beymer. Face recognition under varying pose. In Computer Vision and Pattern Recognition, pages 756761, 1994.

12. Braz, M.R.; Vergilio, S.R., Software Effort Estimation Based on Use Cases, 30th Annual International Computer Software and Applications Conference, 2006. COMPSAC '06, Volume1, pp. 221 - 228, 2006.

13. Zelkowitz, M. V., Perspectives in Software Engineering. ACM Computing Surveys (CSUR), vol. 10, issue 2, June 1978, pp 197-216.

14. ISO/IEC FCD 9126-1.2: Information Technology Software Product Quality. Part 1: QualityModel, draft 1998. 